Check for updates

Cite this: Phys. Chem. Chem. Phys., 2021, 23, 2686

Received 24th November 2020 Accepted 14th January 2021

DOI: $10.1039 / \mathrm{d} 0 \mathrm{cp06113a}$

rsc.li/pccp

\section{The crystallization enthalpy and entropy of protein solutions: microcalorimetry, van't Hoff determination and linearized Poisson-Boltzmann model of tetragonal lysozyme crystals}

\author{
Lorena Hentschel, ${ }^{a}$ Jan Hansen, (D) ${ }^{a}$ Stefan U. Egelhaaf $\left(\mathbb{D}^{\mathrm{a}}{ }^{\mathrm{a}}\right.$ and Florian Platten (D) *ab \\ During a first-order phase transition, a thermodynamic system releases or absorbs latent heat. Despite \\ their fundamental importance, the heat or enthalpy change occurring during protein crystallization has \\ been directly measured only in a few cases, and the associated entropy change can only be determined \\ indirectly. This work provides an experimental determination and theoretical analysis of the dependence \\ of the molar crystallization enthalpy of lysozyme solutions, $\Delta H_{\text {xtal }}$, on the physicochemical solution \\ parameters. Its value is determined directly by isothermal microcalorimetry and indirectly by a van't Hoff \\ analysis of solubility data, which quantitatively agree. This suggests a two-state crystallization process, in \\ which oligomeric intermediates play a minor role. $\Delta H_{x t a l}$ is found to be negative on the order of few \\ tens of the thermal energy per molecule. It is independent of protein concentration and stirring speed, \\ but weakly depends on salt $(\mathrm{NaCl})$ concentration and solution $\mathrm{pH}$. Assuming that crystals are \\ electrostatically neutral, these trends are explained by a linearized Poisson-Boltzmann theory. In addition, the \\ molar crystallization entropy, $\Delta S_{x t a l}$ is analyzed. The dependence of the van't Hoff entropy on salt concen- \\ tration and $\mathrm{pH}$ is captured by the model, complementing the analysis of crystallization thermodynamics.
}

\section{Introduction}

The crystallization of proteins is relevant to many fields of science and technology, ranging from structural biology, pharmacy, to medicine, and inspiring to physics. ${ }^{1}$ In modern structural biology, the functions of proteins are related to their three-dimensional molecular structure. An important technique to determine the protein structure is X-ray crystallography, for which high-quality crystals are required and hence identifying conditions under which crystals grow represents a major bottleneck. $^{2}$ In biotechnology, crystallization is used for batch purification in order to improve storage and stability of enzymes. ${ }^{3}$ The slow dissolution of protein crystals is exploited in protein-based therapeutics for controlled high-dose delivery with sustained release, which allows for treatments of acute and chronic diseases. ${ }^{4}$ In addition, protein crystallization occurs under both native and abnormal conditions in vivo. ${ }^{5}$ Native in cellulo crystallization is a rare process, but occurs in all kingdoms of life. It is related to specific functions, such as storage, protection

\footnotetext{
${ }^{a}$ Condensed Matter Physics Laboratory, Heinrich Heine University, Universitätsstraße 1, 40225 Düsseldorf, Germany. E-mail: florian.platten@hhu.de ${ }^{b}$ Institute of Biological Information Processing (IBI-4: Biomacromolecular Systems and Processes), Forschungszentrum Jülich, Wilhelm-Johnen-Straße, 52428 Jülich, Germany
}

and catalysis. ${ }^{6}$ Non-native crystallization can be induced by small environmental changes or point mutations. It is often associated with pathological conditions, e.g., those relevant for lens opacification ${ }^{7}$ and anemia, ${ }^{8}$ but also occurs due to recombinant gene expression in host cells. Concepts developed in colloid physics have successfully been applied to rationalize certain aspects of protein crystallizations, ${ }^{9}$ including the DLVO theory to describe inter-protein interactions, ${ }^{10-14}$ the colloidal phase behavior of proteins with short-range attractions ${ }^{15-19}$ and the "crystallization slot" to characterize conditions favoring nucleation based on two-body interactions. ${ }^{20-24}$ Moreover, the study of protein crystallization has opened up new vistas on soft-matter concepts, such as the phase behavior of patchy particles ${ }^{9,25}$ and non-classical crystallization pathways. ${ }^{26-29}$ However, despite these efforts, many aspects of protein crystallization are still not fully understood. For example, screening for optimum crystallization conditions often remains a trial-and-error process. ${ }^{30}$ Hence, further attempts to rationalize aspects of protein crystallization are highly desired.

Protein crystallization depends on both thermodynamic and kinetic factors that govern the equilibrium state as well as the path toward equilibrium, that is nucleation and growth, respectively. ${ }^{31}$ Equilibrium properties, such as the (molar) protein solubility $c_{\text {eq }}$ as well as the change of enthalpy $\Delta H_{\text {xtal }}$ and entropy $\Delta S_{\text {xtal }}$ upon crystallization, are used to describe the 
thermodynamics of protein crystallization and reflect the quantities that stabilize protein crystals. Many experimental efforts have been undertaken to explore the dependence of protein solubility on physicochemical parameters, such as $\mathrm{pH}$ and temperature, ${ }^{32-36}$ as well as type and concentration of precipitant. $^{37,38}$ In particular, a correlation between solubility (or crystallization boundary) and second virial coefficient has been observed. ${ }^{12,39-41}$ However, direct experimental determinations of $\Delta H_{\mathrm{xtal}}$ are rare, as noted previously, ${ }^{42,43}$ and its dependence on the solution parameters has as yet not been systematically explored. This is likely due to experimental difficulties associated with the kinetics and thermodynamics of protein crystallization. On the one hand, to be reliably captured by microcalorimetry, the onset and offset of the crystallization process may neither be too fast compared to the initial thermalization of the sample and instrument nor too slow to allow for a significant signal. On the other hand, the absolute value of $\Delta H_{x t a l}$ is typically less than $100 \mathrm{~kJ} \mathrm{~mol}^{-1,42,44}$ and thus difficult to measure. The value of $\Delta H_{\mathrm{xtal}}$ is expected to largely reflect hydrophobic and hydrogen bonding interactions as well as electrostatic protein-protein and protein-salt interactions. The enthalpic (and entropic) contributions of hydrophobic and hydrogen bonding interactions differ in $\operatorname{sign}^{45-49}$ and thus partially compensate each other. As a consequence, depending on the specific protein studied, $\Delta H_{\text {xtal }}$ can be moderately negative or positive or close to zero. ${ }^{42}$

The few studies in which $\Delta H_{\text {xtal }}$ was examined do not lead to a conclusive picture. Calorimetric and van't Hoff enthalpies are equivalent for simple two-state transitions, ${ }^{50}$ which in some cases has been found for biomolecular ion binding reactions. ${ }^{51}$ However, whether complex processes, such as crystallization, can be effectively modelled as two-state transitions is controversial. For two specific conditions, the directly determined values of $\Delta H_{\text {xtal }}$ were shown to agree with the van't Hoff enthalpy, ${ }^{52}$ but arguments raised against van't Hoff analysis of protein solubility data ${ }^{34}$ have still not been dispelled. Moreover, in order to obtain molar quantities which allow for thermodynamic inferences, the calorimetric enthalpy has to be related to the crystallized protein fraction, which needs to be obtained from independent measurements. Yet, this aspect has been ignored in a previous study. ${ }^{53}$ Furthermore, the magnitude of $\Delta H_{\text {xtal }}$ was found to increase with salt concentration, ${ }^{52}$ whereas the opposite trend was predicted theoretically. ${ }^{49}$

Microcalorimetry represents a powerful method to study the thermodynamic properties of individual proteins, ${ }^{44,54}$ such as unfolding, ${ }^{55}$ protein-ligand ${ }^{56}$ or protein-ion binding. ${ }^{57,58}$ However, it has been only scarcely used for studying protein condensation, due to the difficulties mentioned above. Nevertheless, some calorimetric experiments have been performed to study amyloid fibril formation ${ }^{59-62}$ and protein crystallization, ${ }^{52,53,63,64}$ where the latter focussed on lysozyme as a model protein.

Here, we directly measure $\Delta H_{\text {xtal }}$ of lysozyme solutions by combining microcalorimetry with UV-vis spectroscopy to determine the fraction of protein crystallized. The results are found to agree with the van't Hoff enthalpy as determined based on solubility data. We quantitatively and systematically study the dependence of $\Delta H_{\text {xtal }}$ on the physicochemical solution parameters, such as protein and salt concentrations, $\mathrm{pH}$ value, and stirring speed, and explain the observed trends theoretically. We furthermore determine and theoretically explain the salt and $\mathrm{pH}$ dependences of the van't Hoff entropy. This work thus aims for a comprehensive picture of the crystallization thermodynamics of protein solutions and a resolution of the controversial results of previous studies.

\section{Experimental methods}

\subsection{Sample preparation}

Hen egg-white lysozyme powder (Roche Diagnostics, prod. no. 10837059001 , purity $\geq 95 \%$ ), sodium chloride (NaCl; Fisher Chemical, prod. no. $\mathrm{S} / 3160 / 60$, purity $\geq 99.5 \%$ ), and sodium acetate (NaAc; Merck, prod. no. 1.06268, p.a.) were used without further purification. Ultrapure water with a minimum resistivity of $18 \mathrm{M} \Omega \mathrm{cm}$ was used to prepare buffer solutions. The protein powder was dissolved in $50 \mathrm{mM} \mathrm{NaAc}$ buffer solutions, which were adjusted to $\mathrm{pH} 4.0,4.5,5.0$ and 5.4 by adding small amounts of hydrochloric acid. Solution conditions resemble those of our previous work ${ }^{12,23}$ and allow for a comparison with theory. ${ }^{49}$ Protein stock solutions with an initial protein mass concentration $\rho_{\mathrm{p}} \approx 80 \mathrm{mg} \mathrm{mL} \mathrm{m}^{-1}$ were filtered through an Acrodisc syringe filter with low protein binding (pore size: $0.1 \mu \mathrm{m}$; Pall, prod. no. 4611) in order to remove impurities and undissolved proteins. The final protein concentration (typically $65 \mathrm{mg} \mathrm{mL}^{-1}$ ) was determined by UV-vis absorption spectroscopy and/or refractometry. ${ }^{65}$ For some experiments, higher protein concentrations (up to $150 \mathrm{mg} \mathrm{mL}{ }^{-1}$ ) were needed, which were achieved by ultrafiltration, as described previously. ${ }^{65}$ Samples were prepared by mixing appropriate amounts of lysozyme, buffer and salt stock solutions. Sample preparation was conducted at room temperature $(21 \pm 2){ }^{\circ} \mathrm{C}$, above the solution cloud-points. ${ }^{66,67}$ Due to the high salt content, the samples were prone to crystallize and hence injected into the calorimeter immediately after preparation. Most solution conditions were studied three to five times in order to allow for a statistical analysis.

\subsection{Microcalorimetry}

A VP ITC (isothermal titration calorimeter) instrument (MicroCal) consisting of a pair of identical cells was used to monitor the heat signal of crystallizing protein solutions at $20.0{ }^{\circ} \mathrm{C}$. The reference cell was filled with ultrapure water. Before each measurement, the adiabatic shield was cooled $0.5{ }^{\circ} \mathrm{C}$ below the working temperature in order to compensate for the injection heat and thus to allow for reaching the working temperature fast. The freshly prepared sample solution was injected into the sample cell (volume $V=$ $1.4129 \mathrm{~mL}$, according to the producer) of the calorimeter. The syringe was not used to titrate any ligands into the solution, but to seal the calorimeter and to stir the solution at $270 \mathrm{rpm}$, as recommended by the manufacturer to homogeneously dissipate heat and obtain a stable baseline. For a few conditions, explicitly mentioned below, different stirring speeds have been chosen. The instrument started to record the heat signal, once the working temperature was reached a few minutes after sample preparation. 
While the reference cell was continuously supplied with a small power of $5 \mu \mathrm{cal} \mathrm{s}{ }^{-1}$, the instrument measured the differential power $\Delta P$ between the two cells necessary to hold both cells at the same temperature as a function of time. The instrument was operated in the passive feedback mode, allowing for highest sensitivity, as recommended for slow thermal processes. As the sample solution crystallized, heat was released, such that less power had to be provided; i.e., $\Delta P$ became negative and showed an exothermic peak. When the differential power signal reached the baseline again, which typically occurred one or two days after sample preparation, the exothermic reaction was assumed to be completed and the measurement stopped. Subsequently, the crystal-free supernatant was carefully removed from the sample cell and its protein mass concentration $\rho_{\mathrm{f}}$ determined spectrophotometrically. As equilibrium and hence the molar protein solubility $c_{\text {eq }}$ is only reached after months, ${ }^{68}$ typically, $\rho_{\mathrm{f}} \gg c_{\mathrm{eq}} M$ with the molecular weight of lysozyme $M=14320 \mathrm{~g} \mathrm{~mol}^{-1}$. In addition, the UV-vis spectra were inspected for the presence of oligomers or aggregates. Even if small amounts of such species were present in the supernatant, they would scatter light much stronger than the monomers, reducing the transmission and augmenting the measured absorbance. With increasing wavelength, the amount of scattered light diminishes and thus the increase in absorbance becomes weaker, leading to a gradual decrease of the measured UV-vis absorbance with wavelength. ${ }^{69}$ The UV-vis spectra did not indicate the presence of aggregates or oligomers. Afterwards, the crystallized protein sample was removed from the sample cell, usually containing visually observable small crystals. For very similar solution conditions (without stirring), optical microscopy studies revealed the occurrence of (tetragonal) lysozyme crystals. ${ }^{66}$ In the vicinity of the binodal, spherulitic needle-like crystals have been observed as a polymorph. ${ }^{18,70}$ After each microcalorimetric experiment, the sample cell was meticulously cleaned with ethanol, acetone and ultrapure water to completely remove any residuals. ITC experiments with a drifting baseline and those that did not show the characteristic minimum in $\Delta P$ due to an extended period necessary to reach the working temperature were discarded. The instrumental response, especially regarding the accuracy of the power signal, has been validated by measuring the chelation reaction of calcium with ethylenediaminetetraacetic acid (EDTA).

\section{Results and discussion}

At first, the solution conditions that we want to study are localized in the protein state diagram. In a second step, the different methods used to determine the crystallization enthalpy $\Delta H_{\text {xtal }}$ and entropy $\Delta S_{\text {xtal }}$ are discussed and illustrated with examples. Finally, data on the independence of $\Delta H_{\mathrm{xtal}}$ on protein concentration and stirring speed as well as the weak, but systematic dependence of $\Delta H_{\mathrm{xtal}}$ and $\Delta S_{\mathrm{xtal}}$ on salt concentration and $\mathrm{pH}$ value are presented and discussed. Directly and indirectly determined values of $\Delta H_{\text {xtal }}$ quantitatively agree with each other, and the dependence of $\Delta H_{\mathrm{xtal}}$ and $\Delta S_{\mathrm{xtal}}$ on solution conditions is rationalized theoretically.

\subsection{Crystallization conditions in the state diagram of lysozyme solutions}

The state of a protein solution depends on, e.g., molar protein and salt concentrations, $c_{\mathrm{p}}=\rho_{\mathrm{p}} / M$ and $c_{\mathrm{s}}$, respectively. For a systematic microcalorimetric study of isothermal protein crystallization, solution conditions have to be identified (i) under which crystals form and (ii) microcalorimetric experiments can be successfully performed.

As previous theoretical studies indicate that $\Delta H_{\text {xtal }}$ exhibits only a weak temperature dependence, ${ }^{49,71}$ isothermal crystallization is studied at $20.0{ }^{\circ} \mathrm{C}$, which is a common temperature for protein crystallization experiments. ${ }^{68}$ We focus on acidic $\mathrm{pH}$ values ranging from 4.0 to 5.4 , covering the conditions where the solubility as well as number and size of tetragonal lysozyme crystals are maximized, respectively. ${ }^{72-74}$ In addition, theoretical calculations predict a systematic variation of the crystallization enthalpy over this $\mathrm{pH}$ range. ${ }^{49}$

As pointed out by George and Wilson, ${ }^{20}$ optimum conditions for protein crystallization are met when the (net) attractions between the proteins are neither too weak nor too strong. In this case, the second virial coefficient, $B_{2}$, of the solution is in the range

$$
-10 \lesssim B_{2} / B_{2}^{\mathrm{HS}} \lesssim-1,
$$

where $B_{2}^{\mathrm{HS}}$ is the corresponding value of a hard-sphere system. State diagrams ${ }^{66,68,70}$ and solubility experiments ${ }^{33,34,36}$ with moderately concentrated protein solutions $\left(\rho_{\mathrm{p}} \lesssim 100 \mathrm{mg} \mathrm{mL}^{-1}\right)$ point to a minimum salt concentration necessary to induce crystallization. Microscopic observations together with static light scattering experiments and DLVO calculations of $B_{2}$ for acidic lysozyme-salt solutions ${ }^{12,19}$ indicate that net attractions favor crystallization if $c_{\mathrm{s}} \gtrsim 0.3 \mathrm{M}$ (at room temperature). This hints at a link between $B_{2}$ and solubility $c_{\text {eq }}$, as previously suggested. ${ }^{39}$

The second condition, the feasibility of microcalorimetric experiments, is met if protein concentrations are chosen that lead to moderate supersaturations. At hight supersaturation, nucleation starts within minutes, while it can take days to months at low supersaturation levels. ${ }^{1}$ Neither of these cases can be reasonably studied by microcalorimetry. ${ }^{53}$ In the former case, crystallization is faster than thermostatting after sample loading, which, however, is essential due to the weak heat signal associated with protein crystallization (on the order of microwatts ${ }^{52}$ ) in order to avoid spurious effects due to thermal offsets. In the latter case, the instrument and its surroundings have to be thermally stable over a long time period.

Fig. 1 shows the state diagram of lysozyme solutions in the $c_{\mathrm{p}}$ vs. $c_{\mathrm{s}}$ plane at $\mathrm{pH} 4.5$ and $20.0^{\circ} \mathrm{C}$ and indicates the conditions studied in this work. The state diagram contains data on solubility (black filled circles ${ }^{36}$ ) and metastable liquid-liquid phase separation (LLPS, gray filled squares), where the latter have been extrapolated from cloud-point measurements at fixed protein or salt concentration. ${ }^{65,67}$ With increasing $c_{\mathrm{s}}$ both the solubility and the binodal (solid) lines occur at lower values of $c_{\mathrm{p}}$, reflecting the enhanced (net) attractions. In the present work, protein crystallization has been studied by microcalorimetry for the solution compositions marked with an $\times$, 


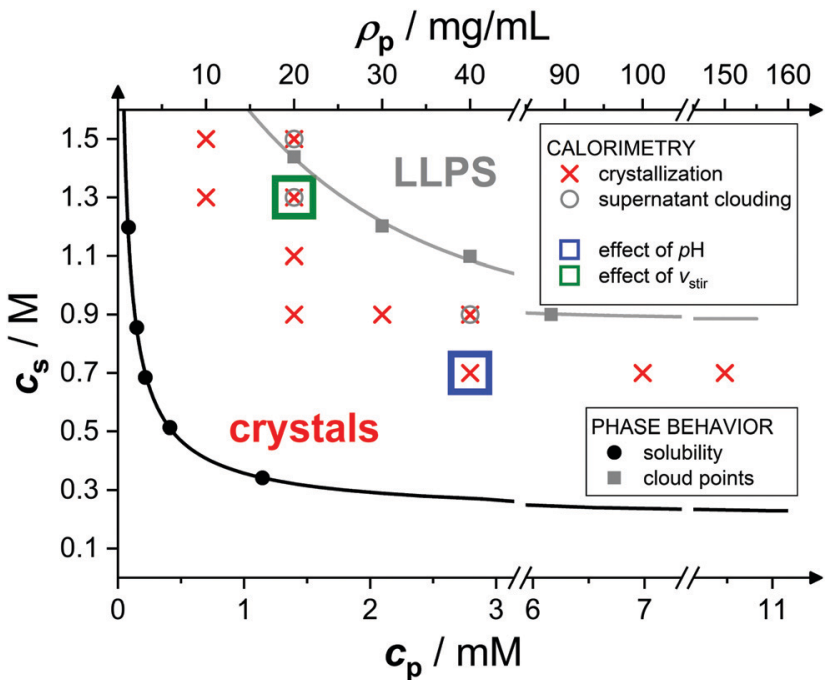

Fig. 1 State diagram of lysozyme solutions at pH 4.5 and $20.0{ }^{\circ} \mathrm{C}$ in the salt concentration $c_{s} v s$. molar protein concentration $c_{p}$ (or, equivalently, mass protein concentration $\rho_{\mathrm{p}}$ ) plane: solubility (black filled circles ${ }^{36}$ ) and cloud-point data (gray filled squares ${ }^{65,67}$ ). Lines are guides to the eye. Microcalorimetric crystallization experiments from the present work, conducted under constant stirring, are marked by an $\times$. Open circles denote that the supernatant was cloudy when removed from the calorimeter and open squares that further solution conditions (as indicated) were investigated.

corresponding to supersaturations $c_{\mathrm{p}} / c_{\mathrm{eq}} \gtrsim 10$. Experiments with smaller $c_{\mathrm{s}}$ or $c_{\mathrm{p}}$ than indicated were not successful. The solutions are investigated under continuous gentle stirring, inducing convection and local shear effects. This might accelerate the crystallization process ${ }^{75,76}$ break large protein clusters, ${ }^{53}$ and displace the phase coexistence curve, ${ }^{77}$ but does not alter the solubility. For some conditions (denoted by a circle symbol), the supernatant was found to be cloudy when removed at the end of the experiment. We speculate that the cloudiness is directly or indirectly related to phase separation. It is, for example, conceivable that the LLPS line is shifted to lower salt concentrations, due to stirring, as observed for colloidal suspensions under shear. ${ }^{77}$ As phase separation is much faster (on the order of seconds $\mathrm{s}^{78,79}$ ) than crystallization, it is expected to occur during the initial equilibration period of the calorimeter (if induced by stirring) and thus to not noticeably affect the microcalorimetric crystallization experiment. In addition, those conditions for which the effects of $\mathrm{pH}$ or stirring speed $v_{\text {stir }}$ have been examined are labelled with a blue and green rectangle, respectively.

\subsection{Determination of the crystallization enthalpy $\Delta H_{\text {xtal }}$ and entropy $\Delta S_{\text {xtal }}$}

The value of $\Delta H_{\text {xtal }}$ can be experimentally determined directly by a combination of microcalorimetry with a spectrophotometric measurement of the protein concentration of the supernatant and indirectly based on a van't Hoff analysis of solubility data. In addition, it can be calculated based on theoretical approaches. The value of $\Delta S_{\text {xtal }}$ is obtained from van't Hoff fits and the model. These different ways will be described and discussed in turn.
3.2.1 Direct microcalorimetric determination of $\Delta H_{\text {xtal }}$. Upon crystallization, binding energy of the protein molecules is released as heat, and the ITC instrument records the temporal evolution of the corresponding exothermal signal. Fig. 2 shows exemplary microcalorimetric data of crystallizing protein solutions $\left(\rho_{\mathrm{p}}=20 \mathrm{mg} \mathrm{mL}^{-1}\right)$ for various salt concentrations as indicated. The curves are qualitatively similar, showing an exothermal peak. The microcalorimetric signal of the solutions with the highest salt concentrations exhibit a steep decrease without initial lag phase. The depth of the minimum in the instantaneous power signal $\Delta P$ increases with $c_{\mathrm{s}}$. The times $t_{\mathrm{i}}$ as well as $t_{\mathrm{f}}$, at which $\Delta P(t)$ starts and ceases to deviate from the baseline, respectively, tend to decrease, while the peak area, i.e., $Q$, seems to be hardly affected. The systematic dependence of $\Delta H_{\text {xtal }}$ on the physicochemical properties of the solution will be discussed in Section 3.3.

The heat $Q$ released upon crystallization is given by the integral of the differential microcalorimetric power signal $\Delta P(t)$, which is limited by $t_{\mathrm{i}}$ and $t_{\mathrm{f}}$ to minimize noise effects:

$$
Q=\int_{t_{\mathrm{i}}}^{t_{\mathrm{f}}} \Delta P(t) \mathrm{d} t
$$

which depends on the volume $V$ of the sample as well as the protein mass concentration $\rho_{\mathrm{p}}$. In few cases, a non-zero, but constant baseline was observed and subtracted from the power signal before performing the integration in eqn (2). Since the initial protein concentration, $\rho_{\mathrm{p}}$, is known and the protein concentration of the supernatant at the end of the microcalorimetric experiment, $\rho_{\mathrm{f}}$, is measured, their difference, $\Delta \rho_{\mathrm{p}}=\rho_{\mathrm{p}}-\rho_{\mathrm{f}}$, can be inferred and $\Delta \rho_{\mathrm{p}} V$ is the crystallized amount of the sample. Hence, the molar enthalpy of crystallization, $\Delta H_{\mathrm{xtal}}$, reads

$$
\Delta H_{\mathrm{xtal}}=\frac{M}{\Delta \rho_{\mathrm{p}} V} Q
$$

The uncertainty of $\Delta H_{\mathrm{xtal}}, \delta\left(\Delta H_{\mathrm{xtal}}\right)$, depends on the uncertainties of the various quantities in eqn (3). The relative

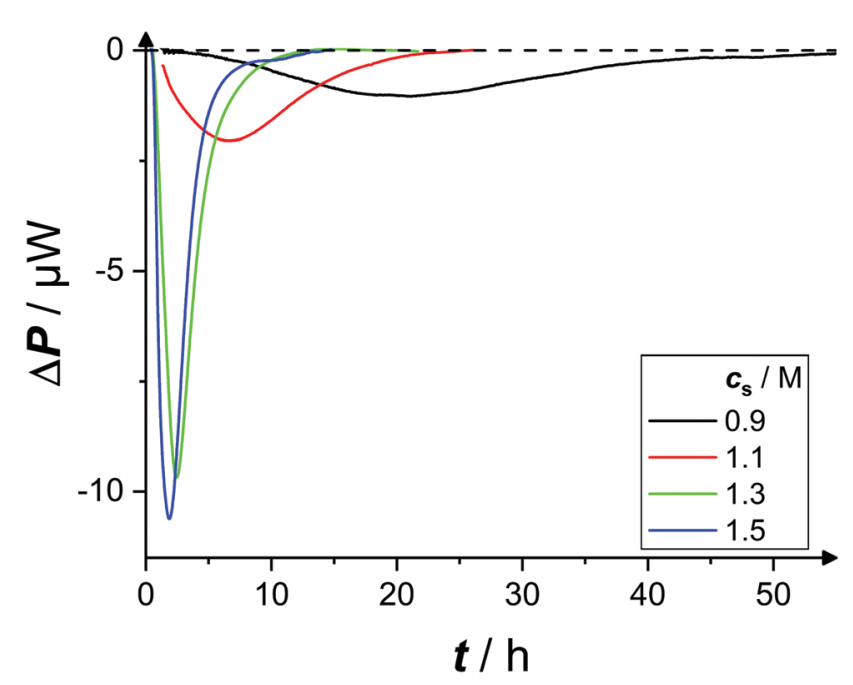

Fig. 2 Exemplary time-dependent microcalorimetric power signals of protein solutions $\left(\rho_{\mathrm{p}}=20 \mathrm{mg} \mathrm{mL}^{-1}, \mathrm{pH}\right.$ 4.5) with various salt concentrations $c_{\mathrm{s}}$, as indicated. 
uncertainty of $\Delta \rho_{\mathrm{p}}$ was calculated, in most cases reaching $\delta\left(\Delta \rho_{\mathrm{p}}\right) / \Delta \rho_{\mathrm{p}}=0.1$, which comprise pipetting and dilution uncertainties of two protein solutions, and that of $Q$ estimated with a typical value $\delta Q / Q=0.05$. Since the relative uncertainties in $M$ and $V$ are at least an order of magnitude smaller, $\delta\left(\Delta H_{\mathrm{xtal}}\right) /$ $\Delta H_{\text {xtal }} \approx 0.1$.

For some solution conditions, in particular at large supersaturations (e.g., $c_{\mathrm{s}}=1.1 \mathrm{M}$ in Fig. 2), the first data point recorded by the instrument might already lie below the baseline. In these cases, the initial part of the recorded signal is extrapolated to the baseline and the associated error in $Q$ estimated to be on the order of the additional area (typically, $\delta Q / Q=0.15$, and sometimes even larger $)$, such that $\delta\left(\Delta H_{\mathrm{xtal}}\right) /$ $\Delta H_{\text {xtal }} \approx 0.2$. The instrument would allow to record the $\Delta P$ signal before the working temperature was reached. However, in this case, the temperature of the sample would be offset, leading to a systematic error in $Q$, which is often neglected in other studies.

3.2.2 van't Hoff enthalpy $\Delta H_{\mathrm{vH}}$ and entropy $\Delta S_{\mathrm{vH}}$ derived from solubility data. A protein molecule will be spontaneously transferred from the solution to a crystal if the associated change in Gibbs free energy is negative: $\Delta G_{\text {xtal }}<0$; i.e., the process is exergonic. The change in Gibbs free energy $\Delta G_{\text {xtal }}$ upon crystallization depends on the enthalpic and entropic contributions, $\Delta H_{\mathrm{xtal}}$ and $\Delta S_{\mathrm{xtal}}$, respectively,

$$
\Delta G_{\text {xtal }}=\Delta H_{\text {xtal }}-T \Delta S_{\text {xtal }}
$$

from which the Gibbs-Helmholtz equation can be deduced:

$$
\frac{\partial}{\partial T}\left(\frac{\Delta G_{\mathrm{xtal}}}{R T}\right)_{p}=-\frac{\Delta H_{\mathrm{xtal}}(T)}{R T^{2}}
$$

with the universal gas constant $R$.

The equilibrium constant $K_{\text {xtal }}$ for the transfer of a molecule from solution to the crystal is defined as

$$
K_{\text {xtal }}=\exp \left(-\frac{\Delta G_{\text {xtal }}^{\ominus}}{R T}\right)
$$

with the standard change of Gibbs free energy upon crystallization $\Delta G_{\text {xtal }}^{\ominus}$ The value of $\Delta G_{\text {xtal }}^{\ominus}$ depends on the choice of the solution standard state; as in ref. 49 , here $c_{\mathrm{p}}^{\ominus}=1 \mathrm{M}$ is the molar protein concentration in a hypothetical solution standard state, in which the proteins are assumed to be non-interacting.

Based on eqn (4) and (6), the logarithm of $K_{\text {xtal }}$ is expected to be a linear function in inverse temperature $T^{-1}$ :

$$
\ln K_{\mathrm{xtal}}=-\frac{\Delta H_{\mathrm{vH}}}{R} T^{-1}+\frac{\Delta S_{\mathrm{vH}}}{R}
$$

where instead of $\Delta H_{\text {xtal }}$ and $\Delta S_{\text {xtal }}$ we use $\Delta H_{\mathrm{vH}}$ and $\Delta S_{\mathrm{vH}}$, respectively, to indicate that here this implicitly refers to the enthalpy and entropy of a two-state transition. Eqn (7) yields the van't Hoff equation:

$$
\Delta H_{\mathrm{vH}}=-R\left(\frac{\partial \ln K_{\mathrm{xtal}}}{\partial T^{-1}}\right)_{p}=R T^{2}\left(\frac{\partial \ln K_{\mathrm{xtal}}}{\partial T}\right)_{p},
$$

which can be derived by considering eqn (5) and (6). (Note that $\Delta H_{\mathrm{vH}}$ is determined under constant (standard) pressure.) This equation implies that if a van't Hoff plot of solubility data, i.e., $\ln K_{\mathrm{xtal}}$ plotted as a function of $T^{-1}$, shows a linear behavior, its temperature-independent slope is related to the van't Hoff enthalpy $\Delta H_{\mathrm{vH}}$ and the heat capacity of the solution does not change between initial and final state. ${ }^{44}$

The value of $K_{\text {xtal }}$ can be determined via

$$
K_{\mathrm{xtal}}^{-1}=\frac{\gamma_{\mathrm{eq}} c_{\mathrm{eq}}}{c_{\mathrm{p}}^{\ominus}} \approx \frac{c_{\mathrm{eq}}}{c_{\mathrm{p}}^{\ominus}}
$$

with the thermodynamic activity coefficients $\gamma_{\mathrm{eq}}$ of the protein in a solution with equilibrium concentration $c_{\text {eq. }}$. For simplicity, the solution is assumed to be close to ideal (i.e., $\gamma_{\mathrm{eq}} \approx 1$ ), which is justified below. As a consequence, the van't Hoff enthalpy can be inferred from the temperature dependence of the solubility.

Despite the amount of protein (typically grams) and time (few months) required for common solubility measurements from batch crystallization, solubility data for tetragonal lysozyme crystals are available in literature for a wide range of temperatures, $\mathrm{pH}$ values and salt concentrations. ${ }^{33-36,70}$ Here, van't Hoff enthalpies are determined based on the comprehensive dataset provided in ref. 36 containing solubility data with two or three significant digits, corresponding to uncertainties ranging from $0.1 \mathrm{mg} \mathrm{mL}^{-1}$ to $1 \mathrm{mg} \mathrm{mL}^{-1}$, for a temperature range (with a temperature control of $0.1 \mathrm{~K}$ ) which covers the temperature used in our calorimetric experiments.

Fig. 3 shows an exemplary van't Hoff plot of the solubility data (open symbols ${ }^{36}$ ) of lysozyme solutions at $\mathrm{pH} 4.5$ for various salt concentrations $\left(2,3,4,5\right.$, and $7 \%(\mathrm{w} / \mathrm{v})$, corresponding to $c_{\mathrm{s}}=$ $0.34,0.51,0.68,0.86$, and $1.2 \mathrm{M})$. For the temperature range available, $1.8 \leq T /{ }^{\circ} \mathrm{C} \leq 22.7$, the natural logarithm of the equilibrium constant exhibits a linear increase with the inverse temperature, and the slopes retrieved from fitting (lines) provide the van't Hoff enthalpy $\Delta H_{\mathrm{vH}}$ according to eqn (8). As expected, $\Delta H_{\mathrm{vH}}$ is negative. The magnitude of the slope and, correspondingly, the value of $\Delta H_{\mathrm{vH}}$ decreases slightly with $c_{\mathrm{s}}$. Further details are discussed in Section 3.3.

In addition, the van't Hoff entropy $\Delta S_{\mathrm{vH}}$ can be inferred from the intercept of a linear fit to the van't Hoff plot according to eqn (7). ${ }^{80}$ (Note that the value of $\Delta S_{\mathrm{vH}}$ depends on the choice of $c_{\mathrm{p}}^{\ominus}$.) The negative intercept of the van't Hoff fit according to eqn (7) in Fig. 3 increases with $c_{\mathrm{s}}$ and, accordingly, $\left|\Delta S_{\mathrm{vH}}\right|$ decreases.

For lysozyme in brine, temperature-dependent virial coefficients are known ${ }^{14}$ and hence activity coefficients can be estimated. ${ }^{81,82}$ As crystallization will only occur if $B_{2}<0, K_{\text {xtal }}$ will generally exhibit smaller values if non-ideality is accounted for by the second virial coefficient. ${ }^{83}$ However, since $B_{2}$ and $c_{\mathrm{eq}}$ are correlated with each other, ${ }^{39}$ i.e., the smaller (more negative) $B_{2}$, the smaller $c_{\text {eq }}$, their effects to non-ideality, to some degree, compensate each other. For the solution conditions considered, the value of $K_{\text {xtal }}$ taking non-ideality into account via $B_{2}$ (filled symbols) increases by a few percent, ranging from $0.14 \%$ (at high $c_{\mathrm{s}}$ and low $T$ ) up to $1.5 \%$ (at low $c_{\mathrm{s}}$ and high $T$ ), compared to the value of $K_{\text {xtal }}$ that assumes ideal 


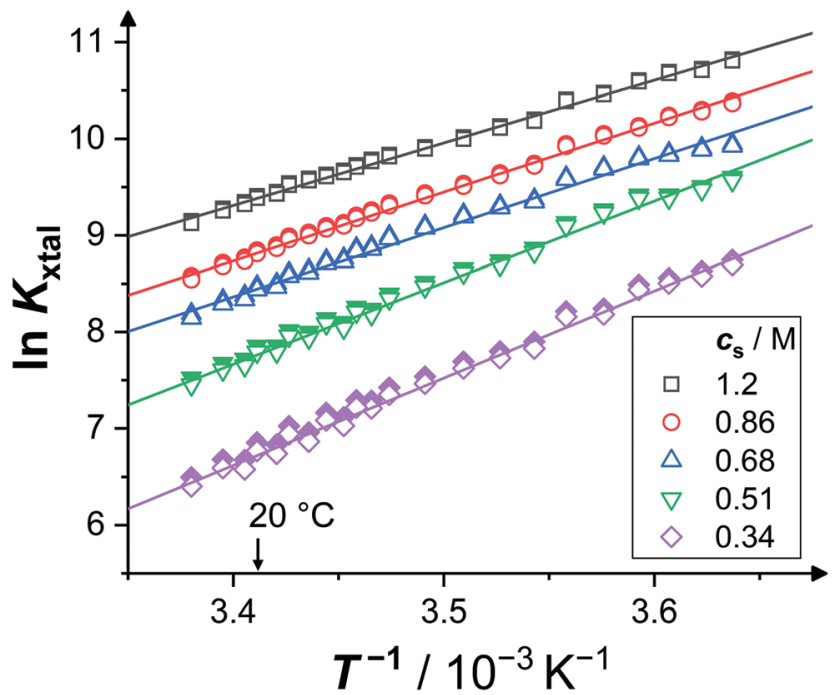

Fig. 3 Exemplary van't Hoff plots: dependence of the equilibrium constant $K_{\text {xtal }}$ on inverse temperature $T^{-1}$ (open symbols; calculated using eqn (9) with solubility data from ref. 36) and corresponding van't Hoff fits (lines), according to eqn (7), for various salt concentrations $C_{s}$, as indicated. Values of the equilibrium constant taking corrections due to non-ideality into account are shown as filled symbols.

solutions (open symbols). However, due to the temperature dependence of the virial coefficients, i.e., $B_{2}$ becomes less negative with increasing $T$ within the relevant range, there is only little effect on the slope of $\ln K_{\mathrm{xtal}}$ : the magnitude of $\Delta H_{\mathrm{vH}}$ (fits not shown) is only very slightly decreased, at most by $2.2 \%$ (at the lowest $c_{\mathrm{s}}$ ) and at least by $0.6 \%$ (at the highest). This small effect is neglected here.

3.2.3 Theoretical model for $\Delta H_{\text {xtal }}$ and $\Delta S_{\text {xtal }}$. Schmit and Dill have developed a model for protein crystallization equilibria. ${ }^{49}$ With parameters adjusted to describe an experimental data set, ${ }^{36}$ it predicts protein solubilities $c_{\text {eq }}$ as a function of temperature, $\mathrm{pH}$ and salt concentration. In Section 3.3, model predictions regarding the crystallization enthalpy are systematically compared with independent experimental data. Protein crystals are considered as electrostatically neutral. Proteins are modelled as spheres of radius $a$ and net charge $Z$ surrounded by a concentric spherical shell of outer radius $b$, which contains water and ions. To achieve charge neutrality, the outer layer is enriched in counterions and depleted in coions as compared to the bulk solution. The values of $a$ and $b$ are set, such that the volume of the compound spheres matches the volume of the unit cell as well as its solvent content; $a=16.1 \AA$, which is close to the linear size of the protein molecule, and $b=19.1 \AA$ for tetragonal lysozyme crystals. $^{49}$

Within this model, the stability of protein crystals is governed by four terms: (i) electrostatic inter-protein repulsion and, more important, counterion sequestration into the crystal, (ii) nonelectrostatic protein-protein contact interactions due to the hydrophobic effect and hydrogen bonding, as well as the (iii) translational entropy of the proteins and (iv) that of counterions.
The two latter (entropic) contributions, (iii) and (iv), oppose crystallization, whereas the two former contributions, (i) and (ii), are enthalpic and favor crystallization. Thus the crystallization enthalpy and entropy read

$$
\begin{gathered}
\Delta H_{\text {xtal }}\left(Z, c_{\mathrm{s}}\right)=\Delta H_{0}+\Delta H_{\mathrm{ES}}\left(Z, c_{\mathrm{s}}\right), \\
\Delta S_{\text {xtal }}\left(Z, c_{\mathrm{s}}\right)=\Delta S_{0}+\Delta S_{\mathrm{ES}}\left(Z, c_{\mathrm{s}}\right)
\end{gathered}
$$

with the temperature-independent non-electrostatic terms $\Delta H_{0}=-49.4 \mathrm{~kJ} \mathrm{~mol}^{-1}$ and $\Delta S_{0}=-95.7 \mathrm{~J} \mathrm{~K}^{-1} \mathrm{~mol}^{-1}$ for tetragonal lysozyme crystals, which were obtained from fits to solubility data in ref. 49 , and the electrostatic terms $\Delta H_{\mathrm{ES}}\left(Z, c_{\mathrm{s}}\right)$ $<0$ and $\Delta S_{\mathrm{ES}}\left(Z, c_{\mathrm{s}}\right)<0$, which depend on the protein net charge $Z$, as set by the $\mathrm{pH}$ value, and the salt concentration $c_{\mathrm{s}}$. The values of $\Delta H_{0}$ and $\Delta S_{0}$ were chosen to account for the positive enthalpy and entropy changes due to hydrophobic burial and the negative enthalpy and entropy changes due to hydrogen bonds in the lysozyme crystals as well as the negative change of internal entropy. ${ }^{49}$

The electrostatic contribution $\Delta H_{\mathrm{ES}}\left(Z, c_{\mathrm{s}}\right)$ can be determined by solving the nonlinear Poisson-Boltzmann equation with appropriate boundary conditions. ${ }^{49}$ Using the linearized (Debye-Hückel) approximation, an analytical expression for the electrostatic enthalpy change has been derived:

$$
\begin{aligned}
\Delta H_{\mathrm{ES}}\left(Z, c_{\mathrm{S}}\right)= & N_{\mathrm{A}} \frac{Z^{2} e^{2}}{4 \pi \varepsilon_{\mathrm{r}} \varepsilon_{0}} \frac{2 \kappa}{(\kappa a+1)} \\
& \times \frac{\mathrm{e}^{-\kappa(b-a)}(\kappa b+1)-(\kappa a+1)}{\mathrm{e}^{+\kappa(b-a)}(\kappa b-1)(\kappa a+1)-\mathrm{e}^{-\kappa(b-a)}(\kappa b+1)(\kappa a-1)},
\end{aligned}
$$

where $N_{\mathrm{A}}$ is Avogadro's number, $\kappa$ the Debye screening constant that depends on the salt concentration, ${ }^{23} e$ the elementary charge as well as $\varepsilon_{0}$ and $\varepsilon_{\mathrm{r}}$ the dielectric permittivity of vacuum and solvent, respectively.

Despite the high charge density of the proteins, both the linear and the non-linear treatment lead to qualitatively similar behavior as a function of $\mathrm{pH}^{49}$ with the main difference being an almost constant offset, i.e., a slightly smaller magnitude of $\Delta H_{\mathrm{ES}}\left(Z, c_{\mathrm{s}}\right)$ in the non-linear case, which could largely be accounted for by readjusting $\Delta H_{0}$. This might, in parts, be ascribed to the relatively high salt concentrations considered here. For simplicity, the analytical expression is used here. To determine the protein net charge $Z$ for a given $\mathrm{pH}$ value, titration data ${ }^{84}$ obtained in the presence of $1 \mathrm{M}$ salt have been interpolated. It is important to note that experimental titration curves ${ }^{84,85}$ are slightly smaller (by about $2 e$ ) at pH 4.0 than the calculated values in ref. 49, which affects $\Delta H_{\mathrm{ES}}$. Eqn (10) and (12) provide a theoretical description of $\Delta H_{\mathrm{xtal}}\left(Z, c_{\mathrm{s}}\right)$ that will be compared with experimental results in the next section.

The electrostatic term $\Delta S_{\mathrm{ES}}\left(Z, c_{\mathrm{s}}\right)$ is determined by the different ions surrounding the protein in the concentric spherical shell. If the protein was uncharged, the total number of ions would be: 
$n=c_{\mathrm{s}} N_{\mathrm{A}} V_{\mathrm{sh}}$ with the shell volume $V_{\mathrm{sh}}=(4 \pi / 3)\left(b^{3}-a^{3}\right)$. The coand counterion enrichment numbers read:

$$
m^{ \pm}=n\left(\exp \left[\mp \operatorname{arsinh}\left(\frac{Z}{2 n}\right)\right]-1\right) .
$$

Thus, $\Delta S_{\mathrm{ES}}\left(Z, c_{\mathrm{s}}\right)$ implicitly depends on $Z$ and $c_{\mathrm{s}}$ :

$$
\begin{aligned}
\frac{\Delta S_{\mathrm{ES}}\left(Z, c_{\mathrm{S}}\right)}{R}= & \left(n+m^{+}\right) \ln \left(1+\frac{m^{+}}{n}\right)-m^{+} \\
& +\left(n+m^{-}\right) \ln \left(1+\frac{m^{-}}{n}\right)-m^{-} .
\end{aligned}
$$

\subsection{Dependence of $\Delta H_{\text {xtal }}$ and $\Delta S_{\text {xtal }}$ on physicochemical solution parameters}

Fig. 4 shows experimental data (symbols) on the crystallization enthalpy for solution conditions differing in stirring speed $v_{\text {stir }}$ and protein concentration $c_{\mathrm{p}}$. The values reported represent weighted averages of the values determined in, typically, three to five individual experiments with independently prepared samples. For the conditions considered, $\Delta H_{\mathrm{xtal}}$ is, within experimental uncertainty, constant (indicated by the dashed lines) at the different stirring speeds and protein concentrations. From a thermodynamic point of view, this is expected, both stirring and protein concentration affect the crystallization kinetics only (not shown). As a consequence of the independence of $\Delta H_{\text {xtal }}$ on $v_{\text {stir }}$, the following experiments have been performed under constant stirring with $v_{\text {stir }}=270 \mathrm{rpm}$ to obtain a reliable measurement. In addition, the enthalpy change upon crystallization determined for solutions with different protein concentrations is averaged taking uncertainties into account.

Fig. 5 shows the resulting dependence of the crystallization enthalpy on salt concentration and $\mathrm{pH}$. Data obtained from direct microcalorimetric measurements (brown filled squares), indirect van't Hoff determinations (blue crosses), literature data (green open symbols, ${ }^{52,53,63,64}$ as indicated) as well as theoretical calculations based on the Schmit-Dill model (red solid lines) are presented. Direct and indirect determinations as well as theoretical predictions yield a consistent picture: within the experimental uncertainties, directly determined and van't Hoff enthalpies quantitatively agree with each other and with the model. The magnitude of $\Delta H_{\text {xtal }}$ tends to slightly decrease with increasing salt concentration and $\mathrm{pH}$ value. According to the theoretical description, this decrease is due to screening of electrostatic inter-protein repulsions at increased $c_{\mathrm{s}}$ or reduced ion sequestration owing to the lower net charge at higher $\mathrm{pH}$. It is important to note that the theoretical model is not a fit to our data, but calculated without any free parameters. The quantitative agreement with the experimental data is therefore particularly significant.

van't Hoff enthalpy data slightly scatter around the microcalorimetric data and the model calculation. Taking interprotein interactions into account in the van't Hoff analysis (see Fig. 3, filled symbols) or restricting the temperature interval of the van't Hoff analysis symmetrically around $T=20^{\circ} \mathrm{C}$ leads to slightly less negative van't Hoff enthalpies, especially at low $c_{\mathrm{s}}$ or
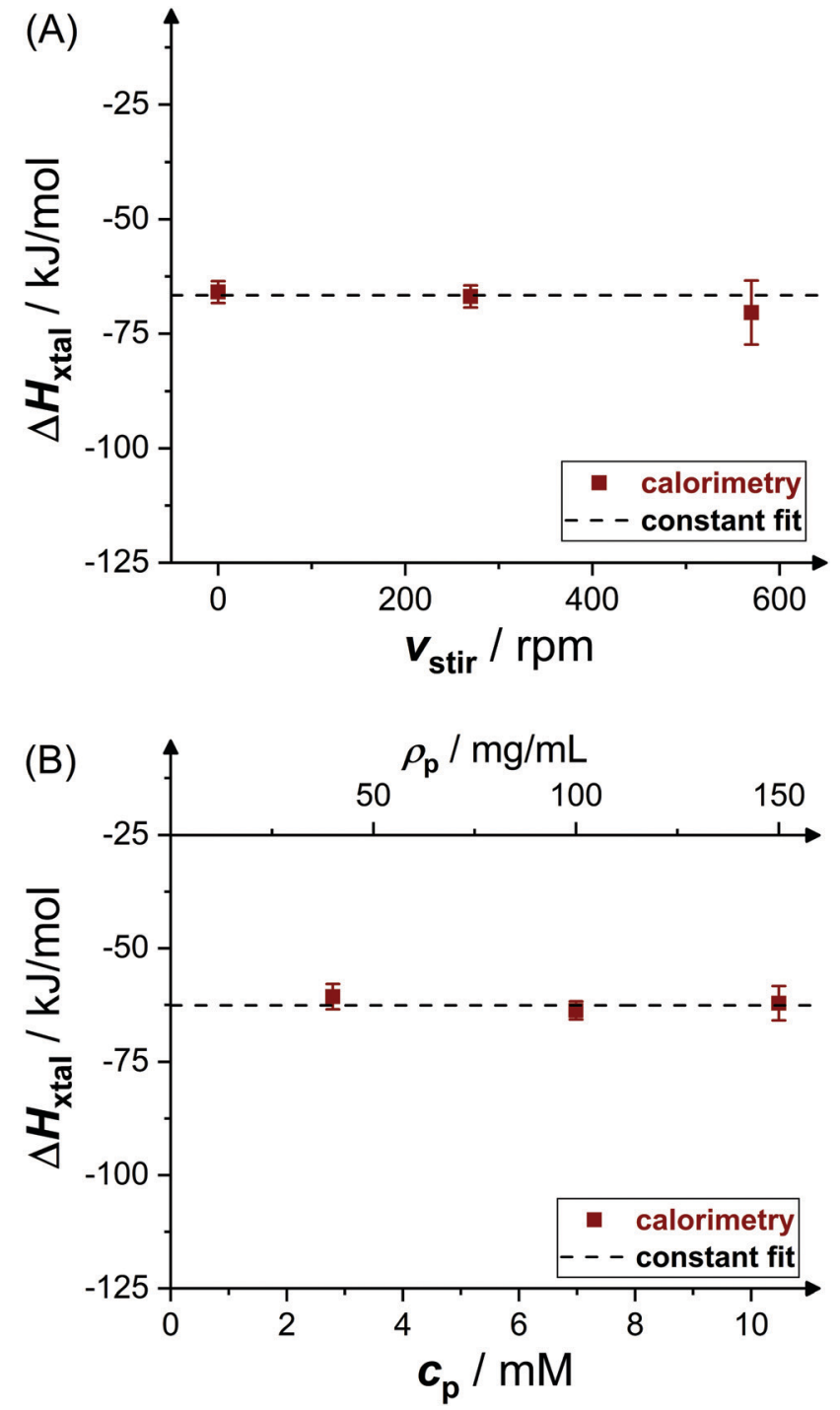

Fig. 4 Effects of (A) stirring speed $v_{\text {stir }}\left(\mathrm{pH} 4.5, \rho_{\mathrm{p}}=20 \mathrm{mg} \mathrm{mL}^{-1}, \mathrm{c}_{\mathrm{s}}=1.3 \mathrm{M}\right)$ and $(B)$ initial molar protein concentration $c_{p}$ or, equivalently, mass concentration $\rho_{\mathrm{p}}\left(\mathrm{pH} 4.5, C_{\mathrm{s}}=0.7 \mathrm{M}\right)$ on crystallization enthalpy $\Delta H_{\text {xtal }}$. Experimental data (symbols) represent averages of several independent measurements.

low $\mathrm{pH}$, and hence an even better agreement (not shown). However, for these solution conditions, either direct measurements have not been successful (low $c_{\mathrm{s}}$ ) or the solubility data show larger uncertainties and scatter more about the van't Hoff fit (low $\mathrm{pH}$ ), thus not allowing for a detailed analysis.

Microcalorimetric literature data grossly scatter around our data. Some values (green hexagons and triangle) follow our trends. ${ }^{52,64}$ In an early experiment (green circle), ${ }^{63}$ a value of $\Delta H_{\text {xtal }}$ with a larger magnitude than observed here was reported. We note that individual experimental runs scatter a lot; both the value and the uncertainty do agree with single runs of our measurement series. In another experiment (green diamonds) $)^{53}$ the heat effect of the solution was considered; i.e., these values have not been normalized by the crystallized fraction of the sample but by the whole solute concentration and, accordingly, have smaller magnitudes. Moreover, their 

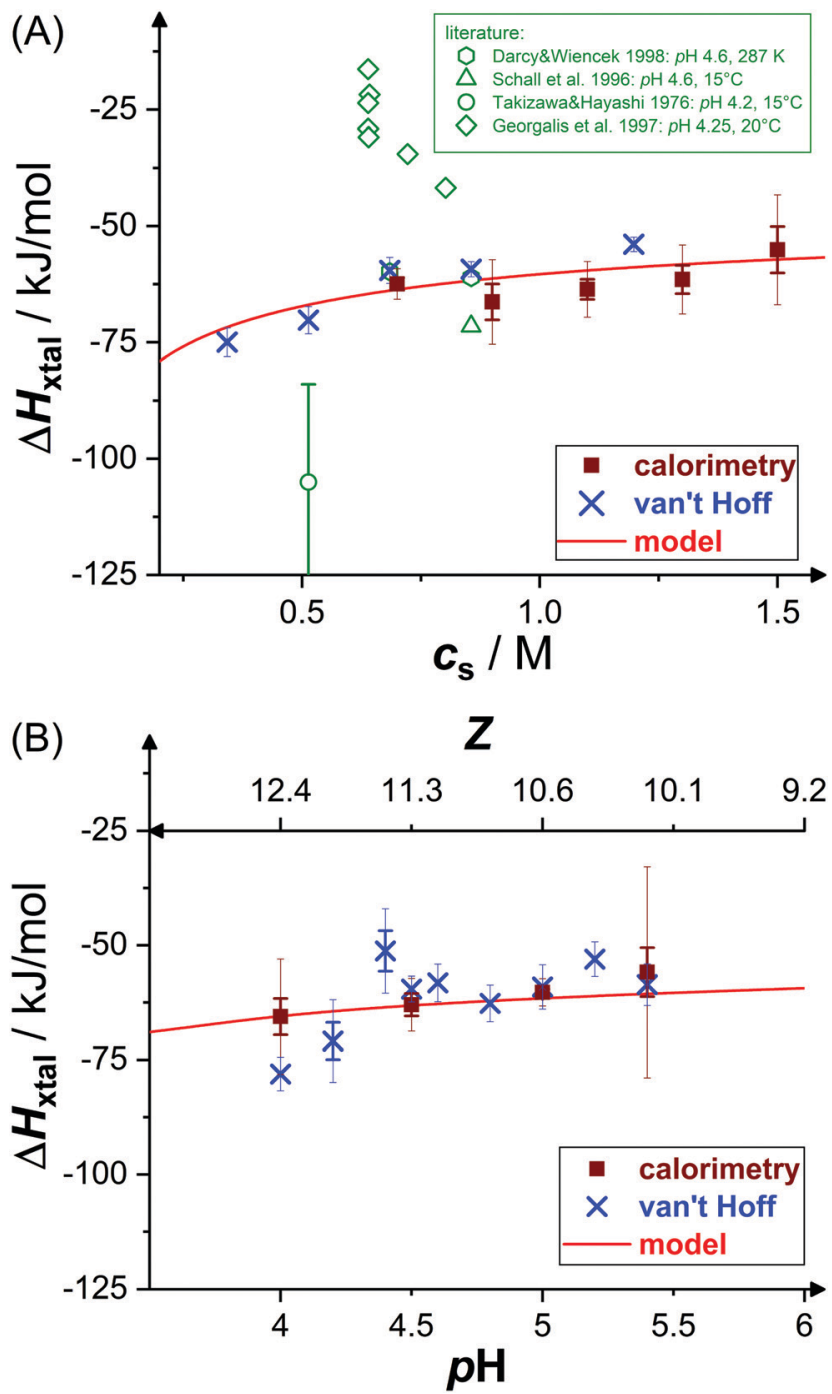

Fig. 5 Effects of (A) salt concentration $c_{s}$ (at $\mathrm{pH} 4.5$ ) and (B) $\mathrm{pH}$ value (or net charge $Z$, at $C_{s}=0.7 \mathrm{M}$ ) on crystallization enthalpy $\Delta H_{\text {xtal }}$ : calorimetric experiments (brown filled squares), van't Hoff enthalpies as calculated from solubility data ${ }^{36}$ (blue crosses), theoretical model ${ }^{49}$ (red solid line: based on eqn (10) and (12)); calorimetric literature data ${ }^{52,53,63,64}$ (green open symbols). Note that some literature data ${ }^{53}$ (green diamonds) were normalized by $\rho_{\mathrm{p}}$ instead of $\Delta \rho_{\mathrm{p}}$. Thin error bars indicate $95 \%$ confidence intervals.

dependence on $c_{\mathrm{p}}$ (at fixed $c_{\mathrm{s}}$ ) or $c_{\mathrm{s}}$ is difficult to compare with our data. Overall, the few previous direct measurements of lysozyme crystallization experiments are well in line with our data or their different results can be rationalized.

Fig. 6 shows the dependence of the entropic contributions to the Gibbs free energy, $-T \Delta S_{\text {xtal }}$, at a temperature of $T=20^{\circ} \mathrm{C}$ on salt concentration and solution $\mathrm{pH}$ value, including indirect van't Hoff determinations (blue crosses) and model calculations (red solid lines). The van't Hoff values range from $31 \mathrm{~kJ} \mathrm{~mol}^{-1}$ to $59 \mathrm{~kJ} \mathrm{~mol}^{-1}$ and, again, slightly scatter around the model calculation. The salt and $\mathrm{pH}$ dependences of $-T \Delta S_{\mathrm{vH}}$ are captured by the model. This indicates contributions to the entropy by the translational entropy of the proteins and ions as well as a constant non-electrostatic contribution.
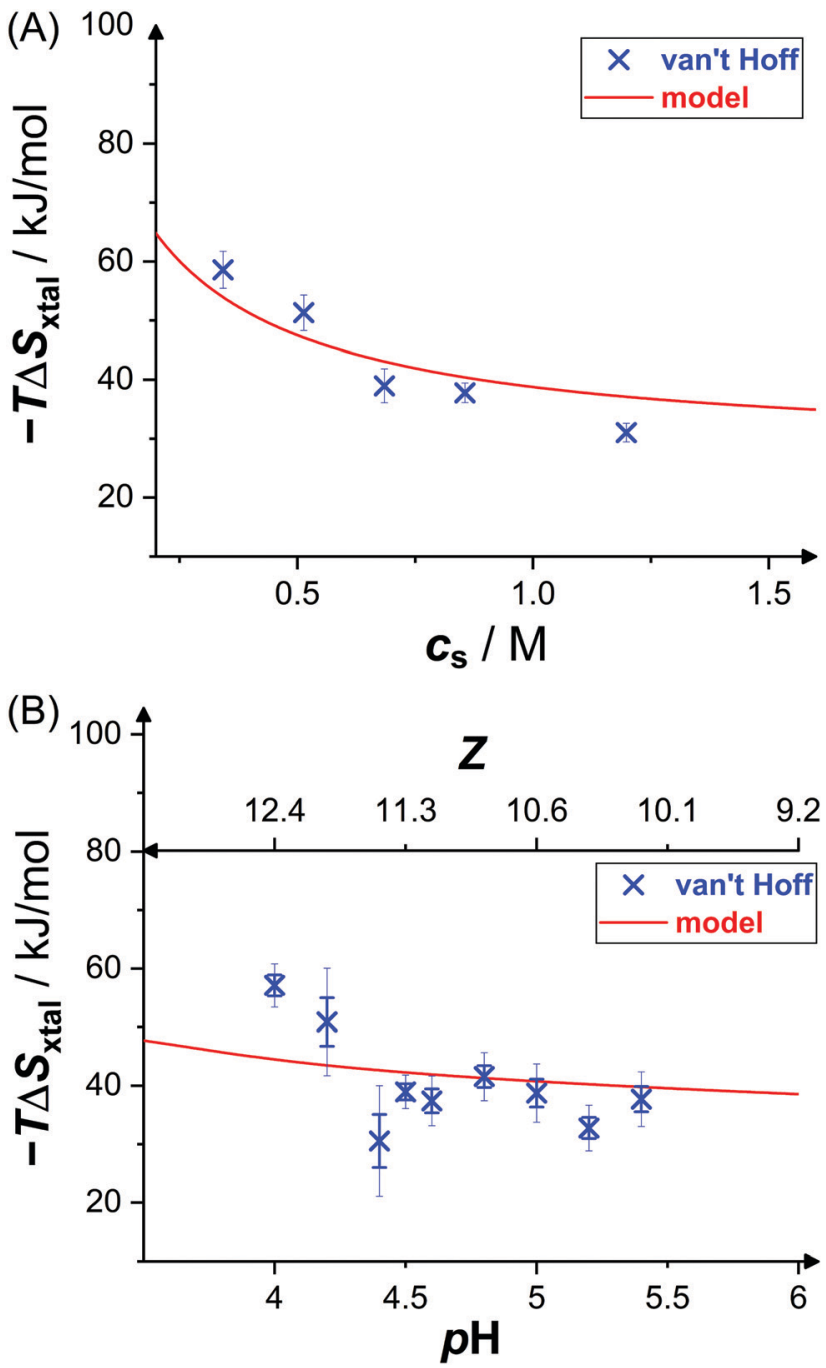

Fig. 6 Effects of (A) salt concentration $c_{s}$ (at $\mathrm{pH} 4.5$ ) and (B) $\mathrm{pH}$ value (or net charge $Z$, at $C_{s}=0.7 \mathrm{M}$ ) on crystallization entropy $\Delta S_{\text {xtal, }}$, shown as its contribution to the Gibbs free energy $-T \Delta S_{x t a l}$ with $T=20^{\circ} \mathrm{C}$ : van't Hoff entropies as calculated from solubility data ${ }^{36}$ (blue crosses) and theoretical model $^{49}$ (red solid line; based on eqn (11) and (14)). Thin error bars indicate $95 \%$ confidence intervals.

Furthermore, according to the model, the standard change of the Gibbs free energy upon crystallization (not shown) ranges from $-17 \mathrm{~kJ} \mathrm{~mol}^{-1}$ to $-22 \mathrm{~kJ} \mathrm{~mol}^{-1}$ (i.e., from $-7 R T$ to $-9 R T$ ) at $T=20^{\circ} \mathrm{C}$ for the conditions studied, which agrees with values computed from solubility data ( $c f$. Fig. 3) using eqn (6).

\subsection{Discussion}

We have carried out and analyzed a number of microcalorimetric lysozyme crystallization experiments, performed a van't Hoff analysis of solubility data and described the experimental observations by a theoretical model. Experimental, van't Hoff and theoretical enthalpy and entropy values semi-quantitatively agree. The analysis of the microcalorimetric experiments reveals that the enthalpy of tetragonal lysozyme crystallization is about $-65 \mathrm{~kJ} \mathrm{~mol}^{-1}$. $\Delta H_{\mathrm{xtal}}$ was neither observed to depend on stirring speed nor on protein concentration, as expected for 
thermodynamic consistency. According to the model, ${ }^{49} \Delta H_{\mathrm{xtal}}$ and $\Delta S_{\text {xtal }}$ are dominated by hydrophobic and hydrogen bonding interactions as well as electrostatics. They weakly, but systematically vary with salt content and $\mathrm{pH}$ value. The decrease of the magnitude of $\Delta H_{\mathrm{xtal}}$ with salt concentration is in contrast to a previous study, ${ }^{52}$ in which the opposite trend was reported. Their claim was based on a van't Hoff analysis of solubility data ${ }^{33}$ that were measured at few temperatures and with lower precision, whereas our van't Hoff analysis (blue crosses in Fig. 5) of the more comprehensive and accurate data $\operatorname{set}^{36}$ revealed the opposite trend in accordance with the direct microcalorimetric experiments. Nevertheless, for the two solution conditions microcalorimetrically probed in ref. $52\left(c_{\mathrm{s}}=0.86 \mathrm{M}, \mathrm{pH} 4.6\right.$ (green triangle in Fig. 5(A)); $c_{\mathrm{s}}=0.51 \mathrm{M}, \mathrm{pH} 5.2$; both at $15{ }^{\circ} \mathrm{C}$ ), a coincidence of microcalorimetric and van't Hoff enthalpies was observed. The agreement of different independent methods in this work provides confidence that the controversial observations regarding the weak salt-dependence of $\Delta H_{\text {xtal }}$ are resolved and underlines the importance of accurate solubility measurements to reach reliable thermodynamic conclusions. The agreement between the van't Hoff and model entropies provides further support.

Here, the agreement between direct and van't Hoff values as well as theoretical predictions has been observed for a broad range of solution conditions and is thus unlikely to be incidental, despite concerns previously raised against van't Hoff analysis of protein solubility data ${ }^{34}$ that have so far not been clarified. The coincidence of direct and van't Hoff enthalpies suggests that the tetragonal lysozyme crystallization process can be effectively described as a two-state transition. Some experiments, ${ }^{86}$ in particular atomic-force microscopy observations, ${ }^{87}$ have suggested that tetragonal lysozyme crystals grow by addition of oligomers, especially tetramers, preformed in the bulk solution. However, comprehensive light scattering experiments did not indicate any evidence for prenucleation aggregate formation. ${ }^{88,89}$ Moreover, the dependence of the growth rate of protein crystals on solution conditions showed best agreement with a model assuming monomer adhesion as mechanism, which includes non-productive binding in wrong orientations to describe the growth rate at high supersaturations. ${ }^{24}$ Furthermore, in our experiments, the UV-vis analysis of the supernatant removed after the microcalorimetric experiments did not indicate the presence of oligomers or aggregates, but of soluble monomers only. In view of these previous and the present results, it is plausible to assume that the initial state of our two-state description of lysozyme crystallization is composed of protein monomers and, as a consequence, tetragonal crystal growth is likely to proceed via the integration of monomers into crystals.

Cacioppo et al. $^{34}$ argue further that, due to a lack of knowledge about or uncertainties in the composition of the solvent within the crystalline phase, the chemical potential of the protein in the crystal might not be constant. However, after their publication the sequestration of ions into the crystal has been accurately measured $^{90}$ and the solvent content can be inferred from crystallographic modelling. ${ }^{91}$ At equilibrium, thermodynamics requires the chemical potential of the protein in the solution to be the same as that of the protein in the crystal in agreement with experiments and theory. After long enough equilibration, the supernatant concentration of crystallizing solutions and solutions with dissolving crystals were found to be equal. ${ }^{68}$ Coarse-grained colloidal descriptions, in which the solvent was modelled as a continuum, semi-quantitatively reproduced experimental protein solubility data. ${ }^{9,40,49}$ In view of these findings, the chemical potential of the protein in the crystal appears to be constant for fixed solution conditions. Thus, the arguments previously raised against the van't Hoff analysis of protein solubility ${ }^{34}$ do no longer appear compelling.

\section{Conclusion}

We have measured the heat-power peaks of crystallizing lysozyme solutions and determined the molar crystallization enthalpy, $\Delta H_{\text {xtal }}$, for solutions differing in protein and salt composition as well as $\mathrm{pH}$ value. The experimental results quantitatively agree with van't Hoff enthalpies inferred from solubility data. This indicates that the crystallization process can be effectively considered as a two-state transition. The dependence of $\Delta H_{\text {xtal }}$ and $\Delta S_{\text {xtal }}$ on salt concentration and $\mathrm{pH}$ is captured by a theoretical model based on charge neutrality of the protein crystals and a linearized Poisson-Boltzmann treatment for the electrostatic contribution to the enthalpy and entropy. These consistent experimental and theoretical findings provide a detailed description of tetragonal lysozyme crystallization thermodynamics. This contributes to our understanding of protein crystallization and can aid the search for optimum conditions for protein crystal growth.

\section{Conflicts of interest}

There are no conflicts to declare.

\section{Acknowledgements}

F. P. thanks the Strategic Research Fund of the Heinrich Heine University (F 2016/1054-5) and the German Research Foundation (PL 869/2-1) for funding.

\section{Notes and references}

1 S. D. Durbin and G. Feher, Annu. Rev. Phys. Chem., 1996, 47, 171-204.

2 N. E. Chayen and E. Saridakis, Nat. Methods, 2008, 5, 147-153.

3 C. P. Govardhan, Curr. Opin. Biotechnol., 1999, 10, 331-335.

4 S. K. Basu, C. P. Govardhan, C. W. Jung and A. L. Margolin, Expert Opin. Biol. Ther., 2004, 4, 301-317.

5 J. P. Doye and W. C. K. Poon, Curr. Opin. Colloid Interface Sci., 2006, 11, 40-46.

6 R. Schönherr, J. M. Rudolph and L. Redecke, Biol. Chem., 2018, 399, 751-772.

7 A. Pande, J. Pande, N. Asherie, A. Lomakin, O. Ogun, J. King and G. B. Benedek, Proc. Natl. Acad. Sci. U. S. A., 2001, 98, 6116-6120. 
8 P. G. Vekilov, A. R. Feeling-Taylor, D. N. Petsev, O. Galkin, R. L. Nagel and R. E. Hirsch, Biophys. J., 2002, 83, 1147-1156.

9 D. Fusco and P. Charbonneau, Colloids Surf., B, 2016, 137, 22-31.

10 M. Muschol and F. Rosenberger, J. Chem. Phys., 1995, 103, 10424-10432.

11 R. Piazza, J. Cryst. Growth, 1999, 196, 415-423.

12 H. Sedgwick, J. E. Cameron, W. C. K. Poon and S. U. Egelhaaf, J. Chem. Phys., 2007, 127, 125102.

13 G. Pellicane, J. Phys. Chem. B, 2012, 116, 2114-2120.

14 F. Platten, J. Hansen, J. Milius, D. Wagner and S. U. Egelhaaf, J. Phys. Chem. Lett., 2016, 7, 4008-4014.

15 D. Rosenbaum, P. C. Zamora and C. F. Zukoski, Phys. Rev. Lett., 1996, 76, 150-153.

16 N. Asherie, A. Lomakin and G. B. Benedek, Phys. Rev. Lett., 1996, 77, 4832-4835.

17 W. C. K. Poon, Phys. Rev. E: Stat., Nonlinear, Soft Matter Phys., 1997, 55, 3762-3764.

18 M. Muschol and F. Rosenberger, J. Chem. Phys., 1997, 107, 1953-1962.

19 F. Platten, N. E. Valadez-Pérez, R. Castañeda-Priego and S. U. Egelhaaf, J. Chem. Phys., 2015, 142, 174905.

20 A. George and W. W. Wilson, Acta Crystallogr., Sect. D: Biol. Crystallogr., 1994, 50, 361-365.

21 A. George, Y. Chiang, B. Guo, A. Arabshahi, Z. Cai and W. Wilson, Methods Enzymol., 1997, 276, 100-110.

22 D. F. Rosenbaum, A. Kulkarni, S. Ramakrishnan and C. F. Zukoski, J. Chem. Phys., 1999, 111, 9882-9890.

23 W. C. K. Poon, S. U. Egelhaaf, P. A. Beales, A. Salonen and L. Sawyer, J. Phys.: Condens. Matter, 2000, 12, L569.

24 J. D. Schmit and K. Dill, J. Am. Chem. Soc., 2012, 134, 3934-3937.

25 J. J. McManus, P. Charbonneau, E. Zaccarelli and N. Asherie, Curr. Opin. Colloid Interface Sci., 2016, 22, 73-79.

26 M. Sleutel and A. E. S. Van Driessche, Proc. Natl. Acad. Sci. U. S. A., 2014, 111, E546-E553.

27 A. Sauter, F. Roosen-Runge, F. Zhang, G. Lotze, R. M. J. Jacobs and F. Schreiber, J. Am. Chem. Soc., 2015, 137, 1485-1491.

28 M. A. Vorontsova, D. Maes and P. G. Vekilov, Faraday Discuss., 2015, 179, 27-40.

29 F. Zhang, J. Phys.: Condens. Matter, 2017, 29, 443002.

30 A. McPherson and J. A. Gavira, Acta Crystallogr., 2014, F70, 2-20.

31 P. G. Vekilov and A. A. Chernov, Solid State Phys., 2003, 57, 1-147.

32 T. Arakawa and S. N. Timasheff, Methods Enzymol., 1985, 114, 49-77.

33 S. B. Howard, P. J. Twigg, J. K. Baird and E. J. Meehan, J. Cryst. Growth, 1988, 90, 94-104.

34 E. Cacioppo and M. L. Pusey, J. Cryst. Growth, 1991, 114, 286-292.

35 F. Rosenberger, S. Howard, J. Sowers and T. Nyce, J. Cryst. Growth, 1993, 129, 1-12.

36 E. L. Forsythe, R. A. Judge and M. L. Pusey, J. Chem. Eng. Data, 1999, 44, 637-640.
37 M. M. Ries-Kautt and A. F. Ducruix, J. Biol. Chem., 1989, 264, 745-748.

38 J.-P. Guilloteau, M. M. Riès-Kautt and A. F. Ducruix, J. Cryst. Growth, 1992, 122, 223-230.

39 B. Guo, S. Kao, H. McDonald, A. Asanov, L. Combs and W. W. Wilson, J. Cryst. Growth, 1999, 196, 424-433.

40 C. Haas, J. Drenth and W. W. Wilson, J. Phys. Chem. B, 1999, 103, 2808-2811.

41 S. Ruppert, S. I. Sandler and A. M. Lenhoff, Biotechnol. Prog., 2001, 17, 182-187.

42 P. G. Vekilov, in Handbook of Crystal Growth, ed. T. Nishinaga, Elsevier, Boston, 2nd edn, 2015, ch. 19, pp. 795-871.

43 Z. S. Derewenda and A. Godzik, in Protein Crystallography, ed. A. Wlodawer, Z. Dauter and M. Jaskolski, Humana Press, New York, 2017, ch. 4, pp. 77-115.

44 P. L. Privalov, Microcalorimetry of Macromolecules: The Physical Basis of Biological Structures, Wiley, Hoboken, 6th edn, 2012.

45 G. I. Makhatadze and P. L. Privalov, Biophys. Chem., 1994, 51, 291-309.

46 P. Ross and M. Rekharsky, Biophys. J., 1996, 71, 2144-2154.

47 J. M. Richardson and G. I. Makhatadze, J. Mol. Biol., 2004, 335, 1029-1037.

48 R. L. Baldwin, J. Mol. Biol., 2007, 371, 283-301.

49 J. D. Schmit and K. A. Dill, J. Phys. Chem. B, 2010, 114, 4020-4027.

50 K. A. Dill and S. Bromberg, Molecular Driving Forces: Statistical Thermodynamics in Biology, Chemistry, Physics, and Nanoscience, Taylor \& Francis, New York, 2010.

51 J. R. Horn, D. Russell, E. A. Lewis and K. P. Murphy, Biochemistry, 2001, 40, 1774-1778.

52 C. A. Schall, E. Arnold and J. M. Wiencek, J. Cryst. Growth, 1996, 165, 293-298.

53 Y. Georgalis, P. Umbach, A. Zielenkiewicz, E. Utzig, W. Zielenkiewicz, P. Zielenkiewicz and W. Saenger, J. Am. Chem. Soc., 1997, 119, 11959-11965.

54 S. Leavitt and E. Freire, Curr. Opin. Struct. Biol., 2001, 11, 560-566.

55 M. Senske, D. Constantinescu-Aruxandei, M. Havenith, C. Herrmann, H. Weingärtner and S. Ebbinghaus, Phys. Chem. Chem. Phys., 2016, 18, 29698-29708.

56 P. C. Weber and F. R. Salemme, Curr. Opin. Struct. Biol., 2003, 13, 115-121.

57 M. Bončina, J. Lah, J. Reščič and V. Vlachy, J. Phys. Chem. B, 2010, 114, 4313-4319.

58 O. Matsarskaia, F. Roosen-Runge, G. Lotze, J. Möller, A. Mariani, F. Zhang and F. Schreiber, Phys. Chem. Chem. Phys., 2018, 20, 27214-27225.

59 J. Kardos, K. Yamamoto, K. Hasegawa, H. Naiki and Y. Goto, J. Biol. Chem., 2004, 279, 55308-55314.

60 M. D. Jeppesen, K. Hein, P. Nissen, P. Westh and D. E. Otzen, Biophys. Chem., 2010, 149, 40-46.

61 A. K. Buell, C. M. Dobson and T. P. Knowles, Essays Biochem., 2014, 56, 11-39.

62 T. Ikenoue, Y.-H. Lee, J. Kardos, H. Yagi, T. Ikegami, H. Naiki and Y. Goto, Proc. Natl. Acad. Sci. U. S. A., 2014, 111, 6654-6659. 
63 T. Takizawa and S. Hayashi, J. Phys. Soc. Jpn., 1976, 40, 299-300.

64 P. A. Darcy and J. M. Wiencek, Acta Crystallogr., Sect. D: Biol. Crystallogr., 1998, 54, 1387-1394.

65 F. Platten, J. Hansen, J. Milius, D. Wagner and S. U. Egelhaaf, J. Phys. Chem. B, 2015, 119, 14986-14993.

66 H. Sedgwick, K. Kroy, A. Salonen, M. B. Robertson, S. U. Egelhaaf and W. C. K. Poon, Eur. Phys. J. E: Soft Matter Biol. Phys., 2005, 16, 77-80.

67 C. Gögelein, D. Wagner, F. Cardinaux, G. Nägele and S. U. Egelhaaf, J. Chem. Phys., 2012, 136, 015102.

68 G. Feher and Z. Kam, Methods Enzymol., 1985, 114, 77-112.

69 S. M. Kelly, T. J. Jess and N. C. Price, Biochim. Biophys. Acta, 2005, 1751, 119-139.

70 Y. Liu, X. Wang and C. B. Ching, Cryst. Growth Des., 2010, 10, 548-558.

71 D. N. Petsev, X. Wu, O. Galkin and P. G. Vekilov, J. Phys. Chem. B, 2003, 107, 3921-3926.

72 M. Ataka and S. Tanaka, J. Phys. Soc. Jpn., 1978, 45, 1779-1780.

73 M. Ataka and S. Tanaka, Biopolymers, 1986, 25, 337-350.

74 R. A. Judge, R. S. Jacobs, T. Frazier, E. H. Snell and M. L. Pusey, Biophys. J., 1999, 77, 1585-1593.

75 A. Onuki, J. Phys.: Condens. Matter, 1997, 9, 6119-6157.

76 J. J. Cerdà, T. Sintes, C. Holm, C. M. Sorensen and A. Chakrabarti, Phys. Rev. E: Stat., Nonlinear, Soft Matter Phys., 2008, 78, 031403.

77 J. K. G. Dhont, Phys. Rev. Lett., 1996, 76, 4269-4272.
78 M. Shah, O. Galkin and P. G. Vekilov, J. Chem. Phys., 2004, 121, 7505-7512.

79 S. Da Vela, M. K. Braun, A. Dörr, A. Greco, J. Möller, Z. Fu, F. Zhang and F. Schreiber, Soft Matter, 2016, 12, 9334-9341.

80 P. G. Vekilov, A. R. Feeling-Taylor, S.-T. Yau and D. Petsev, Acta Crystallogr., Sect. D: Biol. Crystallogr., 2002, 58, 1611-1616.

81 W. G. McMillan and J. E. Mayer, J. Chem. Phys., 1945, 13, 276-305.

82 T. L. Hill, Thermodynamics of small systems, Benjamin, New York, 1963.

83 M. Grant, J. Cryst. Growth, 2000, 209, 130-137.

84 C. Tanford and R. Roxby, Biochemistry, 1972, 11, 2192-2198.

85 D. E. Kuehner, J. Engmann, F. Fergg, M. Wernick, H. W. Blanch and J. M. Prausnitz, J. Phys. Chem. B, 1999, 103, 1368-1374.

86 M. L. Pusey and A. Nadarajah, Cryst. Growth Des., 2002, 2, 475-483.

87 H. Li, A. Nadarajah and M. L. Pusey, Acta Crystallogr., 1999, D55, 1036-1045.

88 M. Muschol and F. Rosenberger, J. Cryst. Growth, 1996, 167, 738-747.

89 F. Rosenberger, P. Vekilov, M. Muschol and B. Thomas, J. Cryst. Growth, 1996, 168, 1-27.

90 P. G. Vekilov, L. A. Monaco, B. R. Thomas, V. Stojanoff and F. Rosenberger, Acta Crystallogr., 1996, D52, 785-798.

91 M. C. Vaney, S. Maignan, M. Riès-Kautt and A. Ducruix, Acta Crystallogr., 1996, D52, 505-517. 